\title{
Female endocrinology - What is it about?
}

\section{Endocrinologia feminina - Do que se trata?}

Dolores Pardini', Ruth Clapauch ${ }^{2}$

$\mathrm{T}$ his edition of the Brazilian Archives of Endocrinology and Metabolism is dedicated to Female Endocrinology, a branch of Endocrinology committed to the study of hormonal regulation of the female reproductive system and its disturbances, from birth to old age. As women represent half of the world population, it is not surprising that Female Endocrinology is so popular among health professionals and women themselves.

Female Endocrinology is a scientific department of the Brazilian Society of Endocrinology (SBEM), together with Andrology (DEFA). The editors of the present issue are all past or current DEFA presidents: RC (2001-2002 and 2003-2004); PMS (2009-2010), and DPP is the present president (2013-2014).

In this edition, an article on how endocrine disruptors may affect female genital tract development (1) opens the discussion on environmental influences on our bodies. Rachel Carlson, who observed reproductive impairment and thus death of birds exposed to pesticides, first described endocrine disruptors in the late 1950s. Nowadays, we know that many of these compounds are persistent and interfere with the endocrine system (2), with estrogen receptor agonism as their first and most commonly described action. Bisphenol A (BPA) is a high production volume monomer used in the manufacture of a wide variety of polycarbonate plastics and resins used as dental sealants and as food packaging liners, all of which are formed from polymerization of BPA. The BPA molecule has structural features that are similar to $17 \beta$-estradiol and other natural estrogenic compounds found in foods (e.g. daidzein in soy products), which give them the ability to bind to estrogen receptors, when present in high enough concentrations.

Estrogen-like influences and weight gain may, in part, explain why the age of puberty is decreasing to less than 10 years old. In this issue, central precocious puberty is addressed (3) in relation to its etiology, diagnosis, and treatment. Precocious puberty may also be a sign of polycystic ovary syndrome (PCOS).

PCOS is a very important chapter of female endocrinology, a disorder of metabolic, as well as reproductive function; common (although not universal) co-morbidities include insulin resistance, hyperinsulinemia, dyslipidemia, hepatic steatosis, and a predisposition to type 2 diabetes and cardiovascular disease. Sixty percent of patients with PCOS are overweight or obese, and even "normal-weight" subjects have relative visceral adiposity and heightened production of inflammatory adipocytokines $(4,5)$.

Hirsutism is another feature of PCOS that represents major discomfort among adolescent and young adults. A brief review on hirsutism diagnosis and treatment is also part of this issue.
'Medical Departament of Endocrinology and Metabolism, Universidade Federal de São Paulo (Unifesp), São Paulo, SP, Brazil ${ }^{2}$ Laboratory for Clinical and Experimental Research on Vascular Biology (BioVasc), Biomedical Center, Universidade do Estado do Rio de Janeiro (UERJ); and Hospital Federal da Lagoa, Endocrinology Sector, Brazilian Health Ministry, Rio de Janeiro, RJ, Brazil

Correspondence to: Dolores Pardini Rua Júpiter 160, ap. 111 01532-030 - São Paulo, SP, Brazil dpardini@uol.com.br

Received on Oct/14/2013 Accepted on Oct/14/2013 
Prolactin disorders may represent a problem in this context, and they are fully addressed in this issue as well.

Moving into the reproductive years, an up-to-date approach on how to assess ovulation in fertility-seeking women and how to discover if an endocrine disorder is affecting fertility is shown in this issue. This is basic knowledge for the endocrinologist who whishes to follow his patients up, and assist them in their reproductive needs. Traditionally, reduced fertility and pregnancy rates have been reported in women with classic CAH. Fertility rates of $60-80 \%$ and $7-60 \%$ have been reported in women with classic SV and classic SW $\mathrm{CAH}$, respectively. In contrast to reduced fertility in classic $\mathrm{CAH}$, pregnancies are commonly normal and uneventful. Thus, fertility, rather than pregnancy rates, seems to be reduced compared with the general population. Subfertility in females with classic $\mathrm{CAH}$ is due to several contributing factors, including androgen excess, adrenal progesterone hypersecretion, consequences of genital reconstructive surgery, secondary polycystic ovaries syndrome, ovarian adrenal rest tumors, and psychosexual factors (6).

In this edition, we also approach hormonal therapy in transsexual people in this issue. The rationale for medical assessment of transsexual patient lies in the fact that this population is at high risk of suicide, selfmutilation, and depression due to lack of healthcare support. Moreover, the real positive effect of hormone therapy on transsexuals quality of life has been demonstrated (7).

\section{REFERENCES}

1. Teeguarden JG, Hanson-Drury S. A systematic review of Bisphenol A "low dose" studies in the context of human exposure: a case for establishing standards for reporting "low-dose" effects of chemicals. Food Chem Toxicol. 2013;62:935-48.

2. Fontenele EG, Martins MR, Quidute AR, Montenegro RM Jr. [Environmental contaminants and endocrine disruptors]. Arq Bras Endocrinol Metabol. 2010;54(1):6-16.

3. Chauhan A, Grissom M. Disorders of childhood growth and development: precocious puberty. FP Essent. 2013;410:25-31.

4. Freemark M. Management of adolescents with polycystic ovary syndrome. J Clin Endocrinol Metab. 2011;96(11):3354-6.

5. Goodarzi MO, Dumesic DA, Chazenbalk G, Azziz R. Polycystic ovary syndrome: etiology, pathogenesis and diagnosis. Nat Rev Endocrinol. 2011;7:219-31.

6. Mouna FM, Mahdi K, Mohamed A. Reproductive outcomes of female patients with congenital adrenal hyperplasia due to 21-hydroxylase deficiency. Indian $\mathrm{J}$ Endocrinol Metab. 2013;17(5):790-3.

7. Bruck JC. [The social background of male to female transsexuals]. Handchir Mikrochir Plast Chir. 2013;45(4):202-6. 